\title{
A Numerical Study On The Performance And Energy Efficiency Of Transport Refrigeration Vehicles In Bangladesh
}

\author{
Md. Mehran Islam¹, Asif Kabir ${ }^{2}$, Arnab Mustafi Arka², Md. Ashiqur Rahman² \\ ${ }^{1}$ Department of Offshore Engineering \\ Bangabandhu Sheikh Mujibur Rahman Maritime University, Bangladesh \\ mehran.naoe@bsmrmu.edu.bd \\ ${ }^{2}$ Department of Mechanical Engineering, \\ Bangladesh University of Engineering and Technology (BUET), Dhaka, Bangladesh, \\ asifkabir121@gmail.com; arnab.mustafi93@gmail.com; ashiqurrahman@me.buet.ac.bd
}

\begin{abstract}
The demand and scope of the transport refrigeration system, as a part of the food cold chain, is likely to increase at a tremendous pace in Bangladesh in the coming years. However, the local transport refrigeration sector is mostly based on retrofitting light-to-medium-duty commercial trucks with refrigerated chambers and emphasizing solely on reducing the chamber temperature to a set value. As a result, performance parameters such as appropriate product storage pattern, clearance within the chamber, airflow distribution, uniformity of temperature, etc. are not maintained following any scientific guideline. The proposed study reports the findings of a Computational Fluid Dynamics (CFD) study on the performance of the local refrigerated transport vehicles, with an aim to propose solutions for improving the existing scenario. Using the knowledge obtained from the mapping of the transport-refrigerated vehicles in Bangladesh, a numerical investigation is carried out to examine the effect of commonly followed product storage and stacking patterns on the refrigeration performance. For the typical Bangladeshi product-loading scenario, it is found that a considerable temperature gradient exists within the chamber due to poor airflow distribution, resulting in hot spots in some locations. The study also includes the examination and comparison of the performance improvement opportunity if standard practices reported in an earlier study is employed. Preliminary results are reported with the ultimate objective of developing a standard product-loading pattern suitable and implementable in the local context.
\end{abstract}

Keywords: Transport Refrigeration, CFD, Energy Efficiency, Cold Chain.

\section{Introduction}

The food cold chain can be defined as "the uninterrupted handling of the product within a low-temperature environment" during the post-production stages such as harvesting, processing, storage, transport, and marketing until it reaches the final consumer [1]. A complete cold chain might have the following elements: bulk cooling, retail cooling, household cooling, and transportation. Bulk cooling refers to the storage of usually large quantities of products and is commonly known as cold storage in Bangladesh. Retail storage refers to the short-term storage of produce during marketing, such as in the supermarkets and super-shops.

The transport segment is a very important part of the cold chain, which can take various forms such as large trucks to small vans to shipping containers. The refrigerated transport system means refrigerated trucks, vans, containers, and ships for transporting fresh produce, meat, dairy products, beverages, medicine, and other temperature sensitive goods. Refrigerated road transport system (also known as reefer vehicles) includes vehicles such as vans and trucks with an insulated body and active refrigeration system for temperature control.

Direct diesel engine-driven or battery powered refrigeration system is typically used in these vehicles. The diesel enginedriven small and often retrofitted refrigerated trucks dominate the refrigerated transport sector in Bangladesh. Local body manufacturers tend to copy the dimensions of imported refrigerated truck's refrigeration chamber and refit 3-5ton capacity trucks with reverse engineered refrigeration chamber. This practice results in huge cost cuts for the investors and as a result, the growth rate of transport refrigeration in Bangladesh has been tremendous in the last few years. In 2007, 49 new refrigerated vehicles were registered while this number was 299 for the year 2017, respectively. That corresponds to an increase of about $500 \%$ over a period of ten years [2]. 
However, at present, this transport sector is continuing without any government oversight or scientific research. The entire transport refrigeration sector in Bangladesh is based on retrofitting, reverse engineering and intuition. This has become a serious concern as product loading pattern, clearance gap, airflow velocity, and temperature, insulation thickness, etc. all play a significant role in keeping the quality of the perishable product being delivered. Researchers have considered temperature as "the single most important factor" which influences the quality of horticultural products [3]. Quality of perishables will be affected even if the product is exposed to adverse temperatures for short and intermittent periods of time [4]. Transportation by trucks is one of the main steps in the distribution chain where the product can spend anywhere from hours to days in Bangladesh. The overwhelming importance of maintaining temperature and proper cooling conditions inside the transport refrigeration trucks can easily be understood.

The present study reports the findings from a series of numerical simulations of such Bangladeshi refrigerated cargo spaces while considering the two most common operating temperature. Loading patterns observed during a previous field mapping [2] have been simulated to display the performance of traditional intuition-based approach adopted by Bangladeshi transport business owners. An improved loading pattern based on the work of European researchers Moureh and Flick [5] has been adopted and dimensionalized considering the small-scale truck size of Bangladesh compared to the trailers or semi-trailers of Europe. The subsequent improvement of airflow and temperature distribution is noted and discussed

\section{Geometry}

For this study, small-scale refrigerated trucks of 3-ton capacity were considered, as these types of trucks are the most common vehicle used in the food cold-chain in Bangladesh (BD).

The refrigerated cargo space, crate/cargo, and evaporator size, and orientation, etc. were modeled according to the field mapping data [2]. The Refrigerated cargo had length, width, and height of $2.95 \mathrm{~m}, 2.18 \mathrm{~m}$, and $1.47 \mathrm{~m}$, respectively. Similarly, the evaporator dimension was $1.4^{*} 0.38^{*} 0.3 \mathrm{~m}^{3}$. For Bangladesh's representative case (termed as BD Cases from here on), the cargo load was simulated to be a rectangular box of dimension $2.03 * 2.09 * 0.88$ and $2.03 * 1.524 * 0.88$ $\mathrm{m}^{3}$, respectively. The geometric set-up considered for the different cases shown in Figs. 1-4.

Finally, for the standard loading pattern based on earlier work for Europe [5], the dimension of a single crate was $0.61 * 0.3 * 0.97 \mathrm{~m}^{3}$. These crates were staked and oriented into 12 columns each containing three crates.

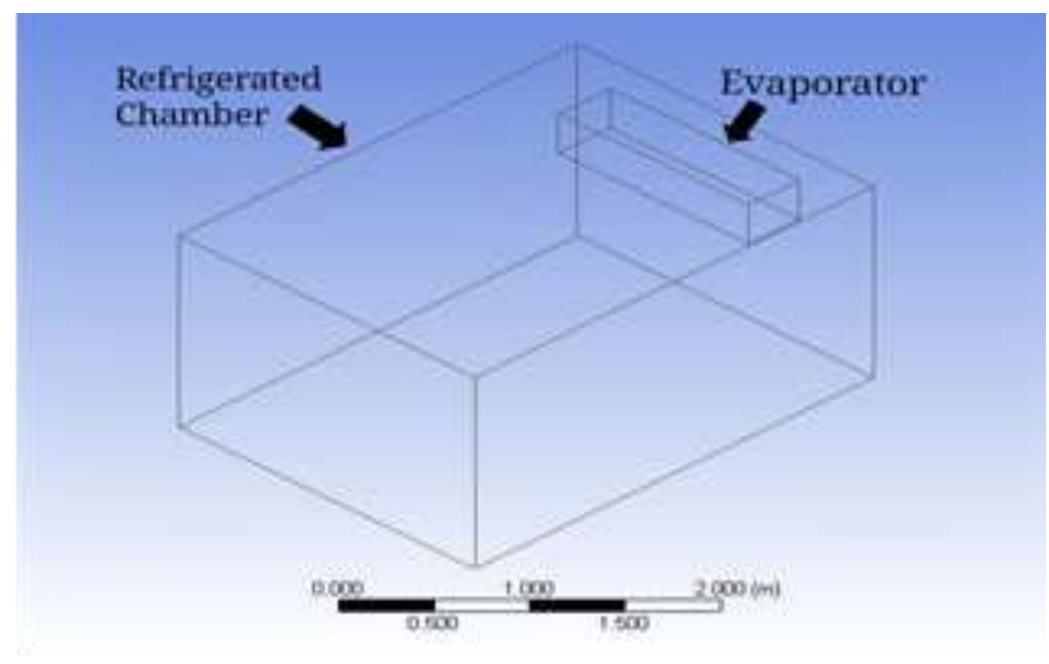

Fig. 1: Geometric set-up of the empty refrigerated chamber. 

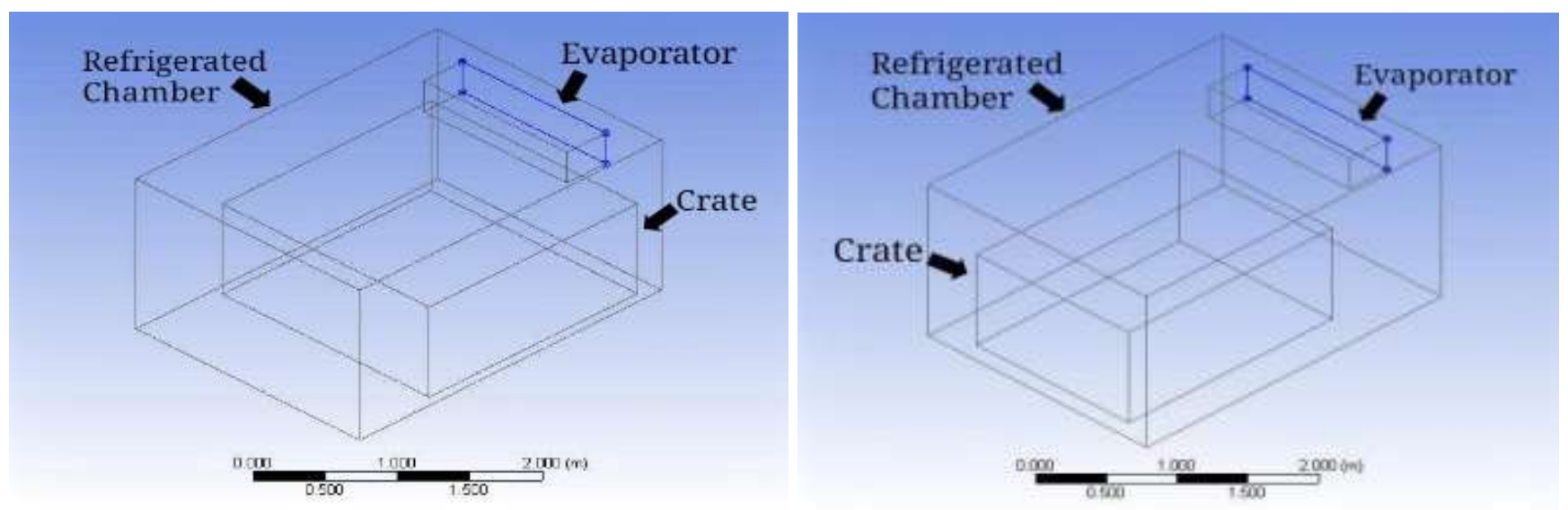

Fig. 2: Geometric set-up of BD storage pattern (a) Case-1 and (b) Case-2.

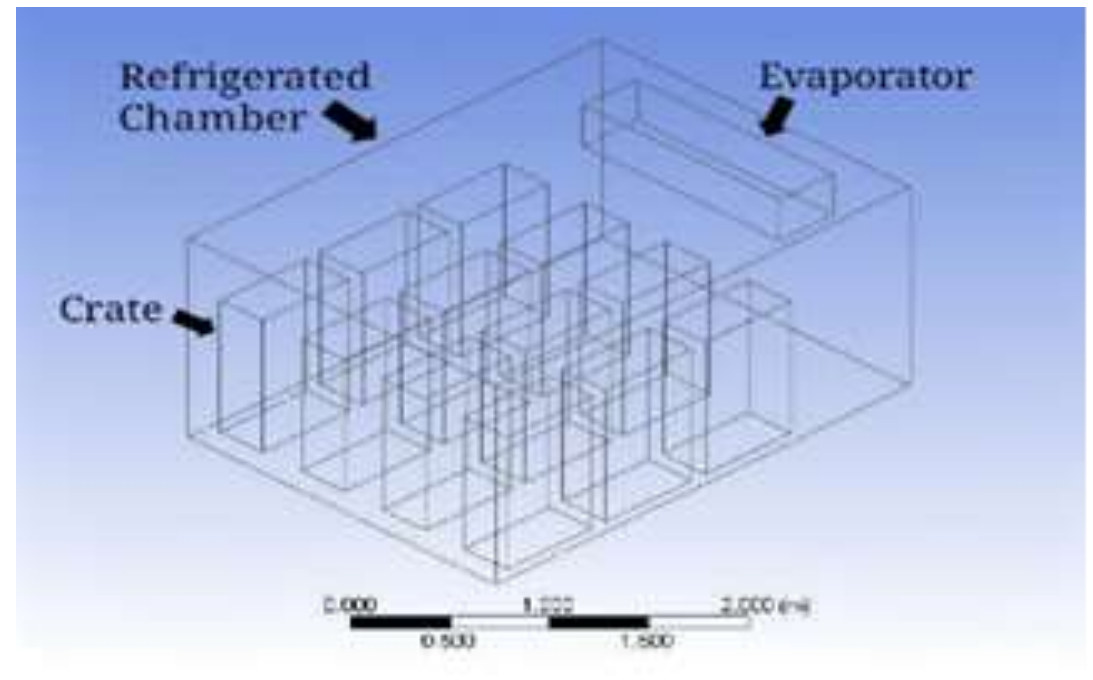

Fig. 3: Geometric set-up of standard storage pattern case as proposed in [5].

\section{Simulation Methodology}

The commercial software package ANSYS Fluent was used for the generation of the model and its subsequent numerical analysis. The governing equations (Continuity equation, conservation of momentum and conservation of energy equation) were discretized by the finite volume technique [6]. Shear stress transport SST k-omega, a modified version of the standard k-omega model, first proposed by Menter [7] was used for turbulence modeling inside the refrigerated chamber. The air inside the refrigerated chamber is assumed to be incompressible fluid. The SIMPLE scheme was chosen for pressure-velocity coupling. Second order upwind scheme was selected for discretizing momentum, the energy equation, turbulent kinetic energy, and specific dissipation rate discretization. The effect of radiation on the outside is neglected as in forced convection applications, radiation is usually discarded. Radiation is also discarded when the surfaces have low emissivity and low to moderate temperature [8]. 
Table 1. Specification of the different cases considered for simulation.

\begin{tabular}{|c|c|c|c|c|c|}
\hline Sl. No. & Simulation information & $\begin{array}{c}\text { Empty } \\
\text { Chamber }\end{array}$ & BD Case-1 & BD Case-2 & Standard Pattern Case \\
\hline 1. & Minimum cell size $(\mathrm{cm})$ & 2 & 2 & 2 & 2 \\
\hline 2. & Maximum face size $(\mathrm{cm})$ & 3 & 3 & 3 & 5 \\
\hline 3. & Maximum size $(\mathrm{cm})$ & 5 & 5 & 186,005 & 193,930 \\
\hline 4. & Nodes & 237,367 & 172,436 & 987,098 & $1,052,536$ \\
\hline 5. & Elements & $1,242,074$ & 902,965 & & 5 \\
\hline
\end{tabular}

A number of different cases were considered for the numerical simulation. The cases considered were: empty refrigerated chamber, a refrigerated chamber with crates loaded having no clearance or gap in between (BD case-1), a refrigerated chamber with crates piled away from the evaporator (BD case-2), and the final case where the crates were organized into separate columns having a gap of $0.27 \mathrm{~m}$ among them (Following the standard storage pattern case as proposed in [5]). Each of these cases was simulated for two different temperature conditions: $268 \mathrm{~K}$ and $253 \mathrm{~K}$. These two operating temperatures were chosen following the findings of previous work [2]. Initially, for all the cases, the refrigerated chamber along with chamber walls was set to the temperature of $300 \mathrm{~K}$. Cold air having a velocity of 7.5 $\mathrm{m} / \mathrm{s}$ and temperatures of $268 \mathrm{~K}$ and $253 \mathrm{~K}$ was simulated to be entering the refrigerated space through the evaporator outlet. A grid independence test was carried out to set a meshing standard for the simulation. The simulation dataset highlighting the results of the grid independence test is given in Table 1.

\section{Results and Discussion}

In the present work, the airflow pattern and the resulting temperature distribution has been studied for refrigerated cargo box usually deployed for commercial transport refrigeration purpose in Bangladesh. As mentioned before, the transport refrigeration sector is completely unmonitored and unstructured in our country. This resulted in a myriad of product staking pattern along with chamber designs that do not comply with any particular set of standard or government approved design.

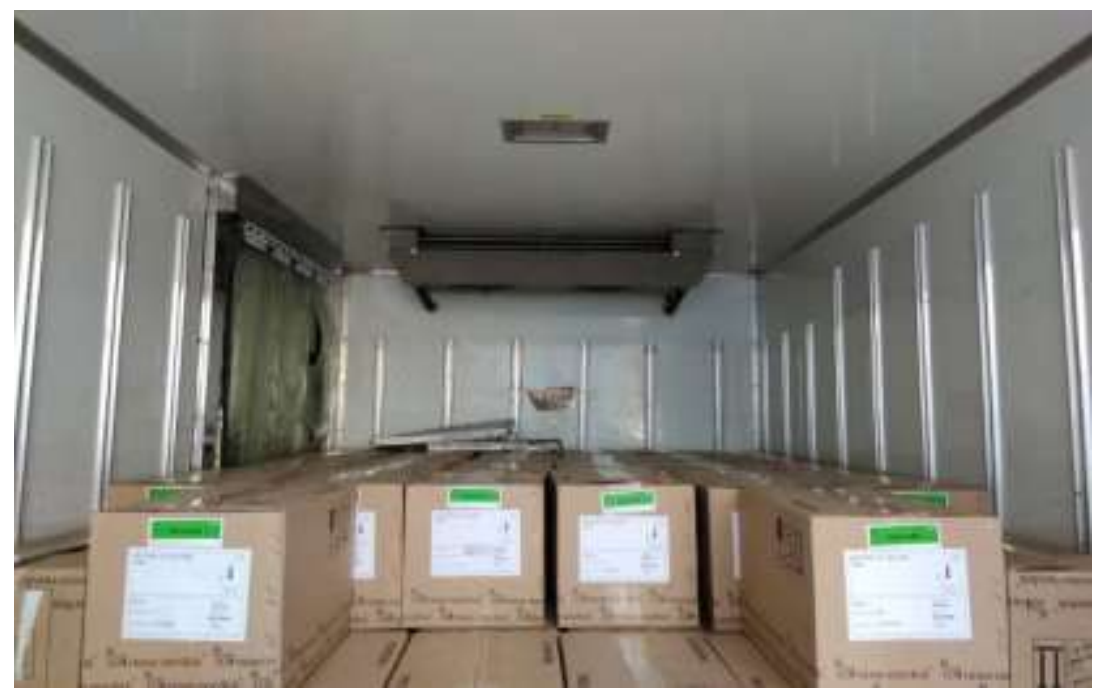

Fig. 5: Typical product loading pattern inside the refrigerated space of the vans as observed during the mapping study [2]. 
In Bangladesh, the loading pattern of crates is based not on the food-shelf life or the quality rather on maximizing the volume of product being delivered [Figure 5]. To reduce the number of trips, the businesspersons stockpile the crates into a cramped condition. This often results in a very little clearance between the crates.

As a result, mapping and designing a unique refrigerated space with one particular set of loading pattern was found to be challenging. Access to such industries and mapping of truck's design, loading pattern, operating temperature, etc. was also proved to be difficult. In the end, this analysis was based on the available data acquired via a detailed field mapping [2]. Two operating temperatures of 268 and $253 \mathrm{~K}$ was chosen along with the dimensions found to be most common during the mapping. Airflow velocity was set to $7.5 \mathrm{~m} / \mathrm{s}$ and it was measured using digital anemometer during the survey.

From the simulation of typical loading pattern scenario of Bangladesh, as shown in Fig. 6, it can be seen that the airflow is significantly reduced and uniform distribution of air between the crates is practically nonexistent. The lack of recirculation is also prominent. These factors result in inconsistent temperature distribution. Especially, away from the evaporator at the backside of the crates, the maximum temperature gradient is observed for the first loading pattern (BD case-1, around $16 \mathrm{~K}$ ). As the top surfaces of the crates are exposed to the airstream, they remain the coldest surface compared to the rest of the crate surfaces signifying the importance of airflow in transport refrigeration temperature management.

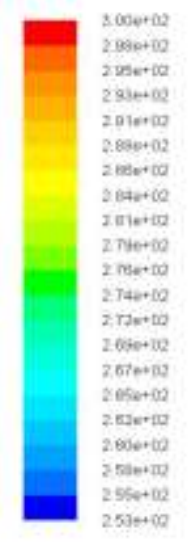

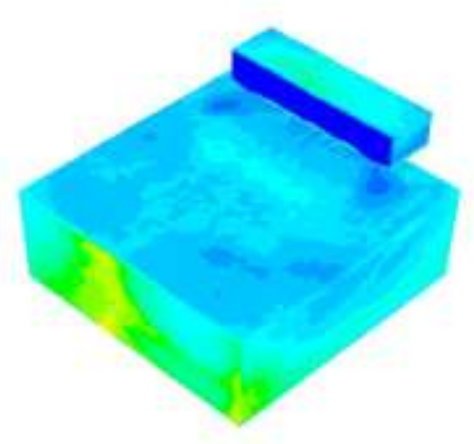

(a)

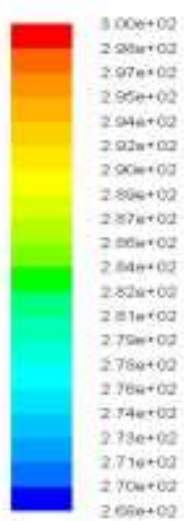

$20 \sin +92$

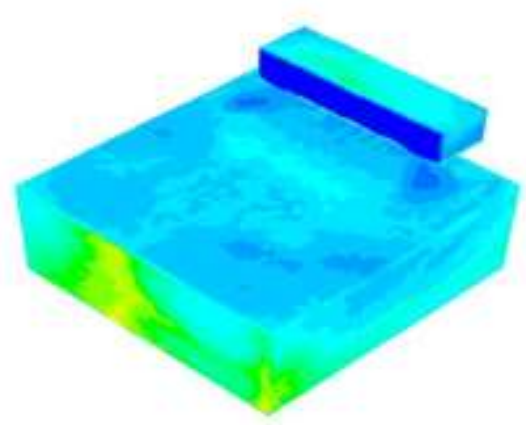

(b)

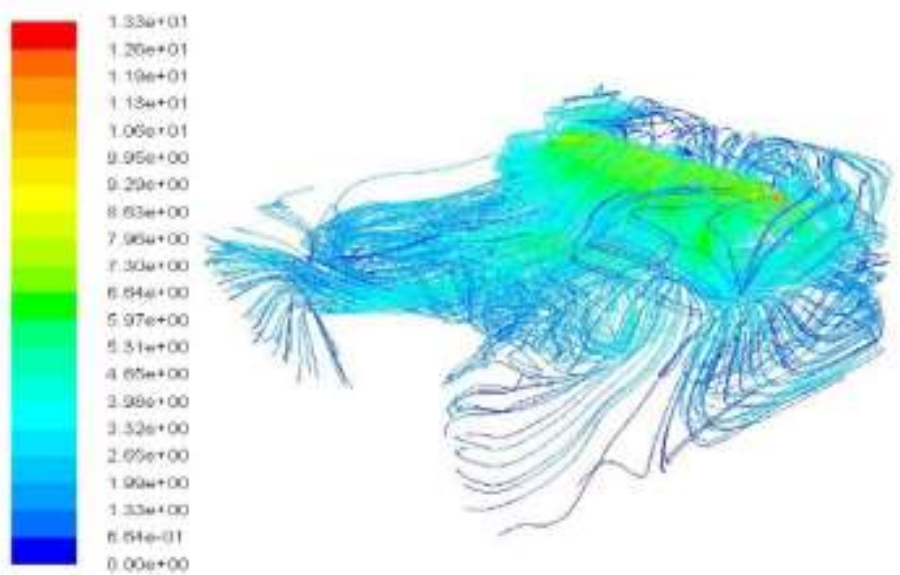

(c)

Fig. 6: For BD case-1 (a) Static temperature distribution around the crates (for $253 \mathrm{~K}$ ), (b) Static temperature distribution around the crates (for $268 \mathrm{~K}$ ), and (c) Airflow inside the chamber.

The poor distribution of storage products may create hot spots inside the chamber in between the cramped crates. While stockpiling results in the maximum amount of products being delivered, it causes the innermost crates in the pile from 
receiving any significant airflow. As it can be seen from the simulation figure, most of the air from the evaporator outlet flows over the crates, hits the back wall of the chamber and re-circulates back to the evaporator.

The second pattern (BD case-2) which was observed during the mapping was the stockpiling of crates away from the evaporator vents and more toward the back door. The people in this trade could not provide any scientific explanation for such an arrangement. However, according to them, they found out that by storing goods in this way, they were able to reduce their product lose and achieve cooling faster than their previous loading attempts. Based on the data received from the personnel involved in this trade in Bangladesh, simulations were carried out and it was found that their assumptions were largely correct. As can be seen from Fig. 7, the airflow pattern is improved compared to the previous case (BD case-1). The temperature gradient at the end of the crate walls was also found to be less than the previous case (a difference of about 11-12 K). This improvement can be attributed to the fact that a smaller number of crates were loaded in the trucks resulting in a reduced volume compared to the BD case-1.

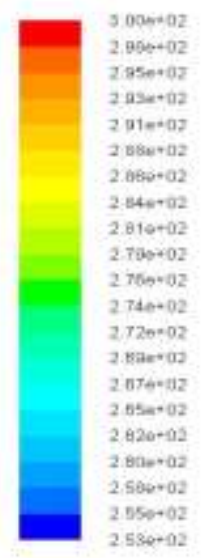

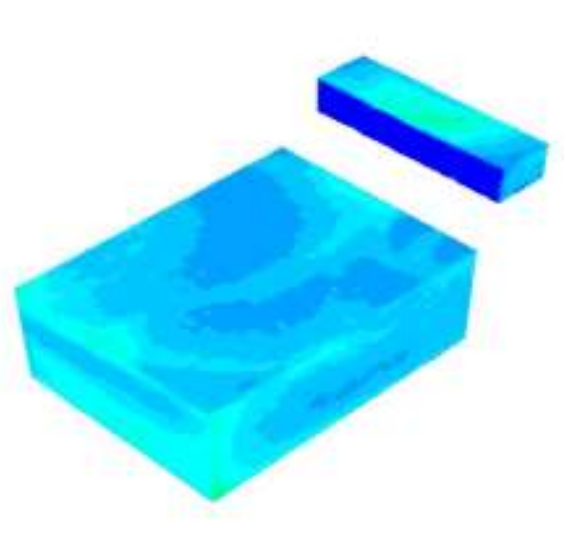

(a)

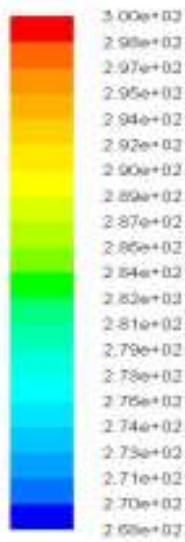

$2600+03$

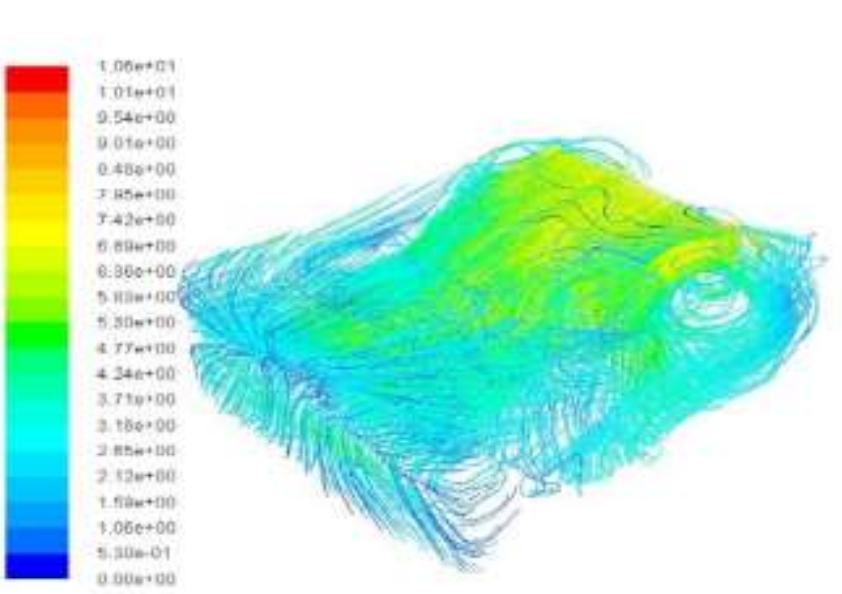

(b)

(c)

Fig. 7: For BD Case-2 (a) Static temperature distribution around the crates (for $253 \mathrm{~K}$ ), (b) Static temperature distribution around the crates (for $268 \mathrm{~K}$ ), and (c) Airflow inside the chamber.

The overall volume reduction in the second case was almost $24.5 \%$ compared to the BD case- 1 . This meant that there was a gap of around $0.7 \mathrm{~m}$ from the sidewall of the chamber to the side walls of the crates. This space was around $0.18 \mathrm{~m}$ for the BD case- 1 . the resulting side gap ensured the recirculation of air and provided overall improved 
temperature distribution. However, in this pattern, the innermost crates of the stockpile were again deprived of cold airflow. These practices may reduce the quality of the products being transported.

In Bangladesh, severe traffic congestions often cause a two-three-hour long trip to stretch for many hours at a time. Such a long journey time and the lack of cooling effect would certainly cause the shelf life of food products to drastically decrease if not causing a total waste of temperature sensitive products. As an improvement to the traditional loading pattern and practice, a new loading pattern based on the work of Moureh and Flick [5] is introduced. For this case, a significant reduction in the volume of cargo being delivered was considered. Individual crates were assembled into a column made of three crates. Twelve such columns were designed. They were separated by a distance of $0.27 \mathrm{~m}$. This resulted in a $40 \%$ volume reduction compared to the traditional Bangladeshi loading pattern (BD case-1).

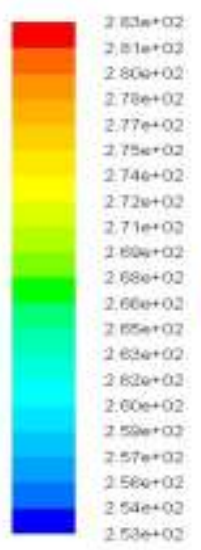

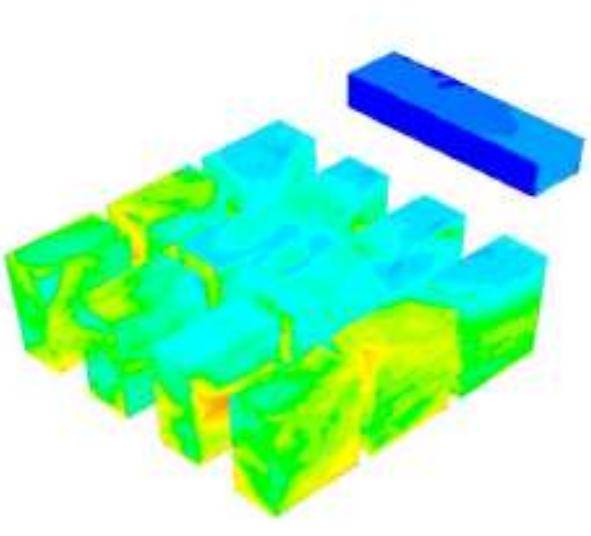

(a)

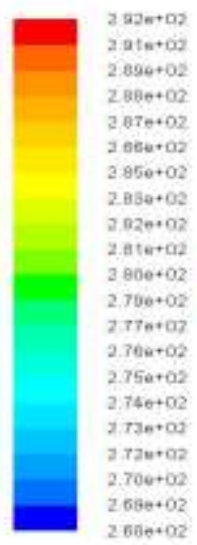

$26 \sin 0+02$

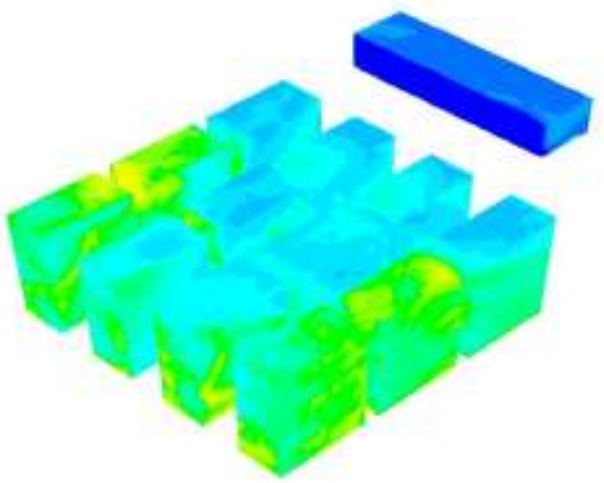

(b)
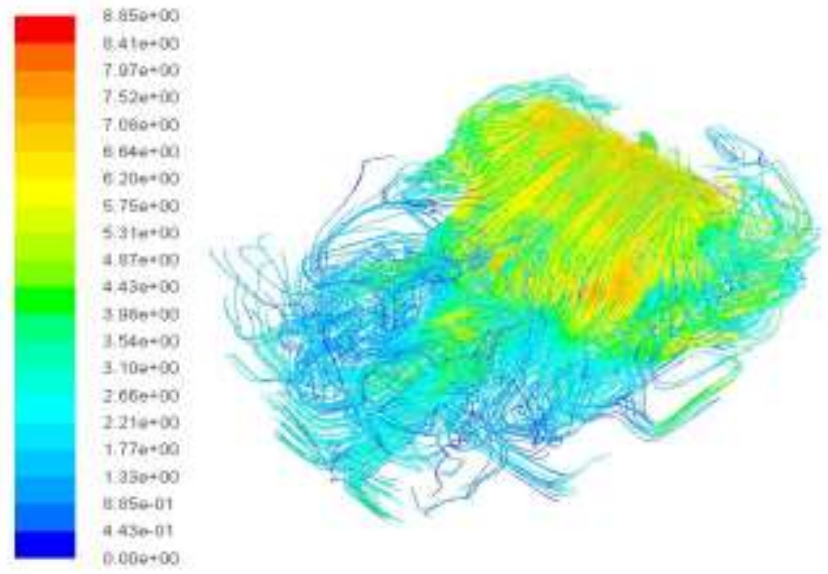

(c)

Fig. 8: For the standard storage loading pattern (a) Static temperature distribution around the crates (for $253 \mathrm{~K}$ ), (b) Static temperature distribution around the crates (for $268 \mathrm{~K}$ ) as per [5], and (c) Airflow pattern for storage loading as per [5].

This spacing helped for more uniform airflow and temperature distribution. As can be seen from Fig. 8, the back wall of the columns placed furthest from the evaporator had temperatures not so high from the evaporator outlet temperature (around $11 \mathrm{~K}$ ). This simple approach of structured loading may significantly improve the product shelf life. Since all the columns are now able to be in contact with the circulating air, the innermost crates of the arrangement had better thermal condition compared to the two BD cases. From the simulation, it can be seen that most of the air flow through the chamber 
center and gradually disperse at the end of the chamber. As a result, columns placed near the center and closest to the evaporator are benefitted from this particular airflow pattern.

There are a number of other techniques that may improve the temperature distribution and airflow conditions further inside a refrigerated chamber. For example, the introduction of T-slotted floors, air chutes, baffles, the introduction of wooden pallets that allow air to be passed beneath the cargo, and plastic curtains, etc. [9,10] have all been shown to be effective measures for ensuring improved thermal conditions inside a refrigerated container. However, these techniques require additional resources and production challenges, which would increase the production cost. These are the prime reasons behind adopting a simple box-shaped chamber by the business owners in Bangladesh. Since the increase in cost and possible reduction in the storage volume would not be easily accepted by the relevant people in the trade and might be unrealistic in the local context, the current study did not consider these techniques as such practices are simply ignored by the local manufacturers

\section{Conclusion}

There remains a clear lack of oversight and knowledge regarding the transport of perishable goods by refrigerated trucks in Bangladesh. The lack of awareness results in un-uniformed temperature distribution throughout the refrigerated chamber, which is amplified by the improper air circulation. These issues are worsened due to the stockpiling of perishable goods instead of storing them in a neat and orderly pattern. The present study reports the performance of the transport-refrigerated vehicles for traditional loading patterns typically followed by the local business owners and compares the findings with the standard product-loading practice as prescribed in an earlier study [5]. The variation of temperature, airflow distribution across the cold chamber and around the stored crates/products were numerically analyzed. Significant improvement was observed for the latter case, although at the cost of the reduction in the maximum storage volume. The preliminary results are reported with further studies in progress for developing a product loading standard appropriate in the local context of Bangladesh.

\section{References}

[1] L.Kitinoja, "Use of cold chains for reducing food losses in developing countries," Population, vol. 6, no. 1.23, pp. 560, 2013.

[2] M. Islam, A. Kabir, A. M. Arka and M. A. Rahman, "Assessment of the Transport Refrigeration System in Bangladesh and Numerical Simulation of the Refrigeration Performance," in 8th BSME International Conference on Thermal Engineering, Dhaka, 2018

[3] R.Wills, B. McGlasson, D.Graham and D.Joyce, Postharvest: an introduction to the physiology and handling of fruit, vegetables and ornamentals. New York: CAB International, 1998.

[4] J.Thompson, F.Mitchell, T.Rumsey, and C. Crisosto, "Commercial Cooling of Fruits, Vegetables, and Flowers," Oakland, C.A.: Division of Agriculture and Natural Resources, University of California, 1998.

[5] J.Moureh and D.Flick, "Airflow pattern and temperature distribution in a typical refrigerated truck configuration loaded with pallets," International Journal of Refrigeration, vol. 27, no. 5, p. 464-474, 2004.

[6] H.K.Versteeg and W.Malalasekera, An introduction to computational fluid dynamics: the finite volume method. Pearson education, 2007.

[7] F. Menter, "Two-equation eddy-viscosity turbulence models for engineering applications," AIAA Journal, vol. 32, pp. 1598-1605, 1994.

[8] Y. A. Cengel and J. Cimbala, Fluid Mechanics Fundamentals and Applications. McGraw-Hill Education, 2014.

[9] "THERMOKING,"[Online]. Available:https://europe.thermoking.com/best-practices/. [Accessed 25 March 2019].

[10] B. H. Ashby, "Protecting perishable foods during transport by motor truck," Agric. Handb. US Dep. Agric., vol. 105, 1970. 